\title{
The effect of noncontingent fixed- and variable-interval reinforcement upon subsequent acquisition of the fixed-interval scallop'
}

\author{
Milton A. Trapold, John G. Carlson and William A. Myers \\ UNIVERSITY OF MINNESOTA
}

\begin{abstract}
Abstraet
Groups of rats received either no pretraining (Group N), pretraining with noncontingent FI-2 min. reinforcement (Group FI), or pretraining with noncontingent VI-2 min. reinforcement (Group VI) prior to learning to bar press on a FI-2 min. reinforcement schedule. The FI scallop was acquired most rapidly by Group FI and least rapidly by Group VI. Moreover, FI pretraining led to faster acquisition of both low post-reinforcement response rates and high pre-reinforcement response rates than VI pretraining.

Problem
\end{abstract}

It has been demonstrated (Bower \& Grusec, 1964) in the free operant situation, that noncontingent discrimination training (training in which, during $S^{D}$, reinforcements are delivered without reference to S's behavior, and during $S^{\Delta}$, no reinforcements are delivered) produces quite large amounts of transfer to a subsequent discrimination task involving the same discriminative stimuli, but in which reinforcements are now contingent upon $\mathrm{S}$ making a specified response. This observation raises the possibility that the acquisition of other behavioral characteristics which are known to develop under particular conditions of response-contingent reinforcement can also be facilitated by prior experiences with the appropriate schedule of noncontingent reinforcement.

The purpose of the experiment reported here was to determine if the scalloped cumulative response curve characteristic of well established behavior on a fixed interval (FI) schedule of reinforcement would be acquired more rapidly if Ss were given extensive prior experience with noncontingent reinforcement on that same FI schedule and less rapidly if Ss were pretrained on a variable interval (VI) schedule.

\footnotetext{
Method

Ss were 18 naive male Sprague-Dawley albino rats approximately 120 days old at the start of the experiment.

The apparatus consisted of two identical automatically programmed Skinner boxes each equipped with a houselight, a removable response lever, and an automatic feeder for delivering $.045 \mathrm{gm}$ food pellets.

Prior to the beginning of the experiment, all Ss were reduced to $80 \%$ of their a d $1 \mathrm{ib}$ weight and then were maintained at that weight throughout the experiment by means of a single feeding of controlled amount after each day's experimental session. Each $\mathrm{S}$ was assigned to one of the two boxes and received all his treatments in that box. All Ss first received one 50-min. adaptation session in the boxes (bar removed), followed by two 25-reinforcement magazine training sessions (bar removed) on which pellets were delivered automatically on a variable interval 2-min. (VI-2) schedule. In the next session, the bar was inserted into the boxes and all Ss were permitted to make 50
}

bar presses, each of which was reinforced. Following this, Ss were divided into three groups of six each (three Ss in each box per group). Group FI then received 12 daily sessions of 25 reinforcements each (bar removed) on which reinforcements were delivered noncontingently on a FI-2 min. schedule. Group VI was treated identically except that reinforcements were delivered on a VI-2 min. schedule (range, $15 \mathrm{sec}$. to $225 \mathrm{sec}$.). Group $\mathrm{N}$ received no treatment during this phase.

Then all Ss were shifted to an FI-2 schedule of reinforcement for bar pressing and run for 15 sessions. Sessions were again 25 reinforcements in length. Fixed interval scalloping was measured by cumulating on separate counters responses occurring in the first and second minute following each reinforcement, and computing for each session a "scallop index" as the ratio of the number of responses in the second minute of the intervals to the sum of the number of responses in the first and second minute.

\section{Results and Diseussion}

The acquisition of the FI scallop for each of the three groups is shown in Fig. 1 where the mean scallop index is plotted against sessions. It is clear from Fig. 1 that although the three groups eventually developed the scallop to the same degree, they did so at quite different rates,.with Group FI acquiring it most rapidly, Group VI least rapidly, and Group $\mathrm{N}$ intermediate. A groups $\mathbf{x}$ trials analysis of variance of the data in Fig. 1 yielded a highly significant groups $x$ trials interaction $(p<.005)$. Using an error estimate based upon all of the data in this overall analysis (Lindquist, 1954, p. 272), the following pattern of .05-level significant differences emerged for the three possible pairwise comparisons among the three groups on each of the 15 days of testing: Group FI

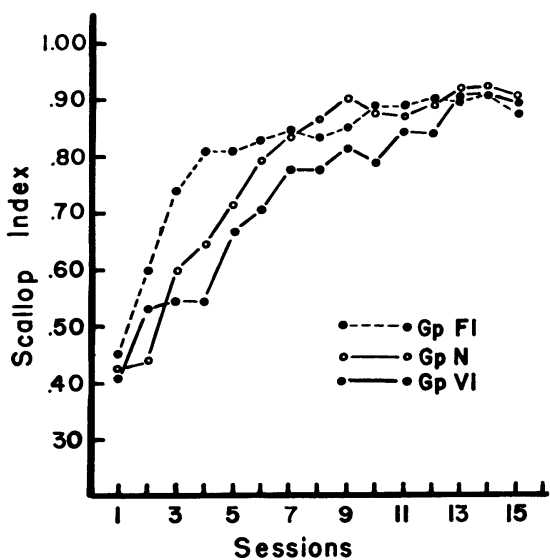

Fig. 1. Mean scallop index as a function of the number of sessions on FI-2 min. reinforcement for Groups FI, VI and N. The scallop index is the proportion of bar presses in a session which occur in the second minute following reinforcement. 


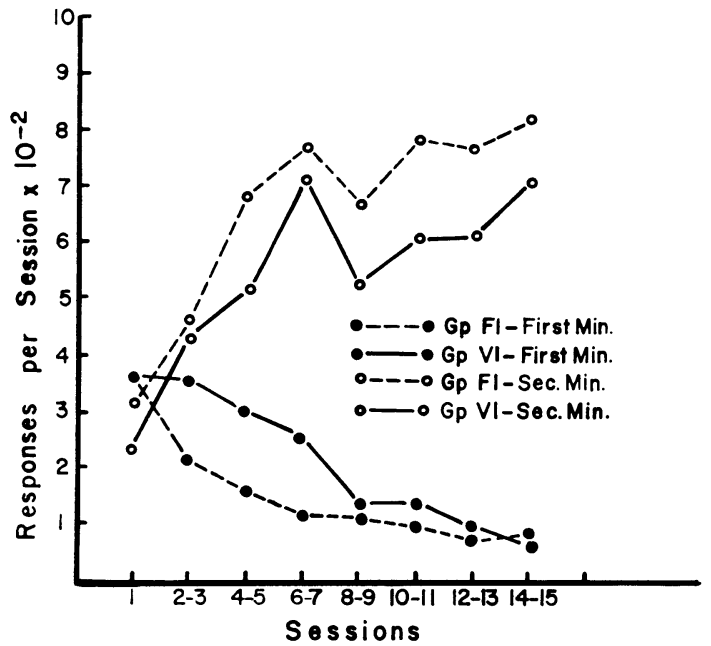

Fig. 2. Mean number of responses per session which occur in the first and in the second minute following reinforcement for Groups FI and VI.

had significantly larger scallop indices than Group VI on days 2 through 5 , and than Group $C$ on day 6 and days 8 through 10 . Group FI had significantly smaller scallop indices than Group $\mathrm{C}$ on days 3 through 5 and on day 10. Thus, the statistical evidence supports the conclusion that noncontingent FI pretraining facilitates and noncontingent VI pretraining retards acquisition of the FI scallop.

The question now arises as to the locus of these effects within the fixed interval. Are they due to differences in the rate of acquisition of low rates in the first half of the interval, high rates in the second half of the interval, or both? Unfortunately, it is impossible to give a complete answer to this question, because Group $\mathrm{N}$ began contingent FI training with response rates in both minutes that were considerably higher than either of the other two groups. This may have been due to the fact that Group N, since it received no noncontingent treatment, had no opportunity to pick up superstitious behavior patterns which could persist into the contingent training phase and compete with bar pressing. Regardless of its explanation, however, this fact makes it impossible to say whether differences in absolute responding between Groups FI and VI are the result of the facilitative influence of FI pretraining, the retarding influence of VI pretraining, or both.

Figure 2 presents first- and second-minute response rates for Groups FI and VI as a function of sessions. It is apparent from Fig. 2 that FI pretraining produced faster acquisition of both low first-minute rates and high second-minute rates, with the former somewhat more predominant early in training and the latter more predominant in later sessions. These impressions were supported by a significant 3-way interaction between Groups, Minutes and Sessions $(p<.01)$. Further analysis of the simple effects, employing an error term based upon the overall analysis and the .05 significance level, yielded the following pattern of differences:
Group FI showed significantly lower first-minute response rates on days 2 through 7 , and significantly higher response second-minute rates on days $1,4,8$ through 13, and 15 .

Thus, there would seem to be little question that noncontingent FI and VI pretraining not only led to different rates of acquisition of the FI scallop, but also had differential effects upon both the first- and second-minute response rates. However, because Group N could not be used as a baseline control, further research will be required to determine the extent to which these differential effects are due to facilitation in the FI condition and/or retardation in the VI condition.

These data, like those of Bower \& Grusec (1964) cannot be interpreted in terms of the direct operation of reinforcement and nonreinforcement upon the strength of bar pressing; the operations which produced differences in the rate of learning in both of these experiments were performed under conditions in which bar pressing could not occur. This conclusion is inconsistent with Dews' (1962) hypothesis that the FI scallop is essentially just a goal gradient phenomenon; i.e., response probability increases with time-since-the-last-reinforcement because the delay of reinforcement is shorter for responses which occur later in the interval. Rather, some form of mediational mechanism would seem to be demanded by these data. One such mechanism, suggested by Bower \& Grusec (1964), assumes that the noncontingent pretraining conditions respondants to the $\mathrm{SD}^{D}$ and $\mathrm{S}^{\Delta}$ (in the present case, these would be unidentified time correlated stimuli), and that these respondants have the capacity to subsequently "modulate" the output of bar pressing. An alternative form of mediational interpretation might point to the possibility that noncontingent pretraining establishes chains of superstitious behavior, and that the chains acquired under noncontingent FI are somehow more facilitative of the development of the FI scallop than those acquired under noncontingent VI (but see Dews, 1962). However, on the basis of available data, it is not possible to draw any firm conclusions as to which of these two mechanisms (for each of which there are numerous logically possible variants), or some other mechanism, is responsible for the transfer observed here. Moreover, it seems unlikely that such conclusions will be forthcoming until much more is known about the ways in which various kinds of noncontingent reinforcement affect learning under conditions of contingent reinforcement.

\section{References}

BOWER, G。, \& GRUSEC, T. Effect of prior Pavlovian discrimination training upon learning an operant discrimination. J. exp. Anal. Behav., 1964, 1, 401-404.

DEWS, P. B. The effect of multiple $S^{\Delta}$ periods on responding on a fixed-interval schedule. J. exp. Anal. Behav., 1962, 5, 369-374. LINDQUIST, E. H. Design and analysis of experiments in Psychology and Education. Cambridge: Houghton-Mifflin, 1953.

Note

1. This research was supported by Research Grant MH-08799-01 from the National Institute of Mental Health to Milton A. Trapold. 